\title{
Ring coupled-cluster doubles correction to geminal wavefunctions
}

\author{
Á. Szabados and Á. Margócsy \\ Laboratory of Theoretical Chemistry, Loránd Eötvös University, \\ H-1518 Budapest, POB 32, Hungary
}

\section{ARTICLE HISTORY}

Compiled February 28, 2017

\begin{abstract}
A ring approximation in coupled cluster $(\mathrm{CC})$ theory is worked out at the multi reference (MR) level. The underlying CC framework is based on generalized normal ordering and applies the corresponding generalized Wick-theorem. Contractions among cluster operators is avoided by adopting a normal ordered exponential Ansatz.

The MR ring CC doubles (MR rCCD) theory is found to represent a companion to the previously introduced extended random phase approximation (ERPA). Equations for wavefunction parameters are derived in parallel and compared. Condition for the ground state consistent with ERPA is obtained. This paves the way towards comparison with the ERPA based correction to the Antisymmetrized Product of Strongly Orthogonal Geminal (APSG) reference, ERPA-APSG.
\end{abstract}

\section{KEYWORDS}

ring coupled-cluster doubles; multireference approach; generalized Wick theorem, random phase approximation; geminal wavefunction

\section{Introduction}

Coupled-cluster (CC) theory, based on a closed shell reference determinant is often relied on when highly accurate quantum chemical results are needed. While the CC parametrization of the wavefunction is in principle exact in the given basis, cutting off the cluster operator is necessary for practical application. Truncation of the cluster operator unfortunately undermines the performance of single reference (SR) CC theory when the so-called static correlation involves determinants beyond the excitation level included in $T$. Such situations call for extension of the original theory e.g. by modifying the amplitude equations[1] or by abandoning the idea of a single, closed shell reference. The latter route has been extensively investigated by professor Debashis Mukherjee throughout his career, starting with open-shell CC formulations[2, 3], followed by ideas based on the eigenvalue independent partitioning $[4,5]$ or those exploiting the JeziorskiMonkhorst[6] parametrization of the wavefunction[7]. An extremely simple version of multireference (MR) CC theory was put forward by Mukherjee and coworkers[8, 9], inspired by the idea of generalizing the concept of normal ordering for a multideterminantal reference function[10,11] It is the internally contracted MR CC formulation of Ref.[8] that provides the starting point of the present work. 
Our focus is now put on the so called ring approximation of the CC doubles equations (rCCD), introduced in the quantum chemical context probably by Cižek[12]. Single reference rCCD theory represents a kind of crossroads of approximation strategies for the molecular electronic structure problem. When formulated with spin-orbitals, equations of the random phase approximation (RPA) can be cast in a form matching the rCCD equations and the rCCD wavefunction can be regarded the ground state consistent with RPA excited states.[13, 14, 15] Admitting several variants, RPA can be derived in the framework of polarization propagator theory[16], it can be obtained in the context of time-dependent (TD) density functional theory (DFT)[17], by combining the adiabatic connection idea with the fluctuation-dissipation theorem within DFT $[18,17]$ or based on Green-function theory.[19] Note, that RPA is understood presently as particle-hole RPA, no consideration of the particle-particle extension is made here.[20, 21]

The ring approximation of CCD is certainly not free from the problems of truncated CC theory mentioned above. As a simple amendment, exchange counterparts of the antisymmetrized integrals can be deleted, leading to the direct rCCD (drCCD) method. The performance of drCCD together with several exchange corrected RPA variants was evaluated by Klopper et al.[22].

When wishing to make rCCD applicable for inherently MR situations, it appears plausible to approach from the multideterminantal reference perspective. Given the various facets of the single reference theory, remarkably diverse routes are provided for a MR extension. Of these possibilities, the RPA approach was picked in several instances. Oddershede, e.g. gave a multireference RPA formulation within the propagator context[16]. Pernal worked out an extended RPA (ERPA) for the multireference situation[23] based on Rowe's equation-of-motion theory[24] for obtaining excited states. Pernal also developped a theory for obtaining a correction to the ground state reference energy based on the solution of the ERPA equations[25, 26].

The present study adds to the picture by exploring the CC aspect in the MR situation. Our goal on one hand is to examine the MR extension of the formal relation between rCCD, RPA and its consistent ground state. A further motivation is provided by our long term interest in applying the antisymmetrised product of strongly orthogonal geminal (APSG) function[27, 28] as a mulideterminantal reference for electron correlation treatment. While a recent study, based on linearized CC, called attention to an inherent failure of the APSG function in the multiple bond breaking situation[29], the success of ERPA-APSG hints that a ring approximation of CC might overcome this problem.

The MR CC method considered here is based on generalized normal ordering (GNO) and applies the corresponding generalized Wick's theorem (GWT) $[9,10,11,30,31]$. Double excitations entering the cluster operator have a general flavour. Contractions among terms of the cluster operator are avoided by assuming a normal ordered exponential Ansatz, suggested by Lindgren[32]. We take an antisymmetrised product of strongly orthogonal geminal (APSG) function[27] as reference and tailor the theory to be as parallel as possible with the existing ERPA-APSG method of Pernal. This is the reason for picking the original rather than the improved version[9] of the underlying CC theory.

In what follows the MR rCCD theory is outlined first, giving explicit formulae within the approximations introduced. A companion RPA theory is presented next and found to agree with Pernal's ERPA equations. The Riccati form of the ERPA equations are contrasted then with the MR rCCD equations. Finally, a set of approximate consistency conditions are derived for ERPA. 
Let us note here, that the ring approximation of SR CCD is not invariant to the way the cluster operator is parametrized, see e.g. the Appendix of Ref.[33]. Equivalence of RPA and rCCD equations as well as strict matching with the consistent ground state of RPA implies a spin-orbital based, closed shell formulation. We work with spin-free operators presently and give the respective relations at the SR level in Appendix.

\section{Preliminaries}

We consider an APSG function[27], denoted by $|0\rangle$, and assume that orthonormal orbitals indexed by $p, q, \ldots$ are natural orbitals of $|0\rangle$. An APSG function is built of two-electron fragments (geminals), each fragment being expanded over a mutually exclusive set of natural orbitals (termed geminal subspaces). The second quantized expression of APSG reads

$$
|0\rangle=\prod_{I=1}^{N / 2} \sum_{p \in I} c_{p} a_{p_{\beta}}^{+} a_{p_{\alpha}}^{+}|\mathrm{vac}\rangle
$$

where $N$ stands for the number of electrons and $I$ refers to the geminal subspace. From Eq.(1) it is apparent, that APSG is expanded in the seniority zero subspace[34] of the Hilbert space. In the case where each geminal subspace is two dimensional, APSG agrees with the generalized valence bond function.

In terms of coefficients $c_{p}$, diagonals of the spin-dependent density matrix are given as

$$
n_{p}=\left\langle 0\left|a_{p_{\sigma}}^{+} a_{p_{\sigma}}\right| 0\right\rangle=c_{p}^{2} .
$$

The corresponding hole density matrix element is denoted by overline:

$$
\bar{n}_{p}=1-n_{p} .
$$

Besides 1-body density matrices, 2-body reduced density matrix elements will be involved in the following development. For completeness, we give here the expression of the 2-body cumulant of APSG, defined as

$$
\lambda_{p_{\sigma} q_{\sigma^{\prime}}}^{r_{\sigma} s_{\sigma^{\prime}}}=\left\langle 0\left|a_{r_{\sigma}}^{+} a_{s_{\sigma^{\prime}}}^{+} a_{q_{\sigma^{\prime}}} a_{p_{\sigma}}\right| 0\right\rangle-\delta_{p r} \delta_{q s} n_{p} n_{q}+\delta_{\sigma \sigma^{\prime}} \delta_{p s} \delta_{q r} n_{p} n_{q} .
$$

Summing for both spin indices of the above spin-dependent cumulant one obtains

$$
\Lambda_{p q}^{r s}=\sum_{\sigma \sigma^{\prime}} \lambda_{p_{\sigma} q_{\sigma^{\prime}}}^{r_{\sigma} s_{\sigma^{\prime}}}=2 \delta_{I_{r} I_{s}} n_{r} n_{s}\left(\delta_{p s} \delta_{q r}-2 \delta_{p r} \delta_{q s}\right)+2 \delta_{I_{p} I_{r}} c_{p} c_{r} \delta_{p q} \delta_{r s}
$$

In the above, $I_{r}$ denotes that geminal subspace which accommodates natural orbital $r$.

A GNO, denoted by $\{$.$\} will be used below, satisfying the condition$

$$
\langle 0|\{.\}| 0\rangle=0 \text {. }
$$

A second quantized string of operators in GNO involves particle and hole density matrices as well as cumulants of the reference function, as described by Kutzelnigg and 
Mukherjee[11] and proved by Kong, Nooijen and Mukherjee[31]. The normal ordered form of the electronic Hamiltonian accordingly reads

$$
H=E_{0}+\underbrace{\sum_{p q} f_{q}^{p}\left\{E_{p}^{q}\right\}}_{\left\{H_{1}\right\}}+\underbrace{\frac{1}{2} \sum_{p q r s} v_{r s}^{p q}\left\{E_{p q}^{r s}\right\}}_{\left\{H_{2}\right\}}
$$

with the APSG reference energy being

$$
E_{0}=\langle 0|H| 0\rangle,
$$

the spin-summed single excitation operator defined as

$$
E_{p}^{r}=\sum_{\sigma} a_{r \sigma}^{+} a_{p \sigma}
$$

and the doubly exciting, spin-summed operator taking the form

$$
E_{p q}^{r s}=E_{p}^{r} E_{q}^{s} .
$$

The two-electron integral in $\langle 12 \mid 12\rangle$ convention is expressed as $v_{r s}^{p q}=\langle r s \mid p q\rangle$ and the Fockian entering Eq.(5) reads

$$
f_{q}^{p}=h_{q}^{p}+\sum_{r} \bar{v}_{q r}^{p r} n_{r}
$$

where

$$
\bar{v}_{q r}^{p r}=2 v_{q r}^{p r}-v_{r q}^{p r}
$$

\section{Ring CCD based on APSG}

With the aim of correcting APSG by CC, a cluster operator involving solely double excitations is written as

$$
T_{2}=\frac{1}{2} \sum_{\substack{p<r \\ q<s}} t_{r s}^{p q}\left\{E_{p q}^{r s}\right\}
$$

In the above and throughout index restriction $p<r$ is used as a shorthand for $n_{p} \geq$ $n_{r}, p \neq r$. (Note, that assuming orbitals ordered in decreasing occupation number, $r<p$ is still possible if $n_{p}=n_{r}$. The case of $n_{p}=n_{r}$ is allowed only for non integer occupation numbers. Both $n_{p}=n_{r}=0$ and $n_{p}=n_{r}=1$ are excluded.) Amplitudes $t_{r s}^{p q}$ exhibit the single symmetry relation $t_{r s}^{p q}=t_{s r}^{q p}$. Amplitudes $t_{r s}^{p q}$ and $t_{r s}^{q p}$ are unrelated when $r \neq s$ and $p \neq q$.

A normal ordered exponential Ansatz is taken for the corrected wavefunction

$$
\Psi=\left\{e^{T_{2}}\right\}|0\rangle,
$$


satisfying the intermediate normalization condition

$$
\langle 0 \mid \Psi\rangle=0
$$

by virtue of definition (4) of GNO. Energy $E$ corresponding to $\Psi$ is conveniently written as

$$
E=E_{0}+\Delta E
$$

Substituting Eqs.(5), (7), (8) and (9) in the Schrödinger equation

$$
H \Psi=E \Psi
$$

and projecting from the left by $\langle 0|$ results in the energy correction

$$
\Delta E=\left\langle 0\left|\left\{H_{2}\right\}\left\{T_{2}\right\}\right| 0\right\rangle .
$$

To obtain the amplitude equations, Eq.(10) is projected from the left by the doubly excited functions

$$
\langle 0|\left\{E_{v u}^{y x}\right\}^{\dagger}=\langle 0|\left\{E_{x y}^{u v}\right\}, \quad u<x, v<y .
$$

This results

$$
\begin{aligned}
\left\langle 0\left|\left\{E_{x y}^{u v}\right\}\left\{H_{2}\right\}\right| 0\right\rangle & +\left\langle 0\left|\left\{E_{x y}^{u v}\right\}\left(\left\{H_{1}\right\}+\left\{H_{2}\right\}\right)\left\{T_{2}\right\}\right| 0\right\rangle \\
& +\frac{1}{2}\left\langle 0\left|\left\{E_{x y}^{u v}\right\}\left\{H_{2}\right\}\left\{T_{2}^{2}\right\}\right| 0\right\rangle^{\prime}=0
\end{aligned}
$$

where the prime on the last term on the lhs of Eq.(13) indicates omission of contraction patterns producing $\Delta E$, i.e. where $\left\{H_{2}\right\}$ is contracted completely with one of the $T_{2}$ 's.

Up to this point the derivation followed SR CCD theory $[35,36]$ closely. Index restrictions, e.g. in Eq.(7) taking the place of occupied/virtual categorization and $\left\{\exp T_{2}\right\}$ standing in Eq.(8) instead of $\exp \left\{T_{2}\right\}$ are the only notable deviations. A marked difference with SR theory appears when expectation values in Eqs.(11) and (13) are evaluated based on the GNO and the associated GWT. With ordinary normal ordering, pair contractions (i.e. rank-1 contractions) appear solely in expectation values with the reference. In the general situation contractions up to rank- $k$ appear when calculating the expectation value of a string composed of $2 k$ operators. The geminal structure of the reference represents an advantage at this point. Due to the fact, that 3 -body or higher cumulants (up to $N$ ) are explicitly zero with a geminal reference, at most rank-2 contractions appear in our case. ${ }^{1}$ Even by this, the number of patterns obtained by the GWT is huge, as reflected in the second row of Table 1 . The SR case is shown for comparison in the first row. The couple of hundred terms of the SR case are usually dealt with by diagram techniques. Diagrammatics in the context of GNO and GWT was initiated by Kutzelnigg and Mukherjee[11], it is however less used. When relying on the GWT, contraction patterns are most often generated and processed by computer. [38, 39] We adopt the latter practice, utilizing a code written in our laboratory in PYTHON language.

\footnotetext{
${ }^{1}$ It is interesting to observe that $(N+1)$-body and higher cumulants become nonzero even if the corresponding density matrix is zero[37]. The contribution of these cumulants is neglected presently.
} 
Table 1. Number of contraction patterns, obtained by the GWT, contributing to the terms of the CCD equations, Eq.(13). Cumulants of order $(N+1)$ or higher are omitted, $N$ denoting the number of electrons.

\begin{tabular}{lcccr}
\hline & $\left\langle\{E\}\left\{H_{2}\right\}\right\rangle$ & $\left\langle\{E\}\left\{H_{1}\right\}\left\{T_{2}\right\}\right\rangle$ & $\left\langle\{E\}\left\{H_{2}\right\}\left\{T_{2}\right\}\right\rangle$ & $\left\langle\{E\}\left\{H_{2}\right\}\left\{T_{2}^{2}\right\}\right\rangle$ \\
\hline all patterns, SR CASE & 4 & 16 & 80 & 576 \\
\hline MR CASE & & & & \\
\hline all patterns $^{\mathrm{a}}$ & 41 & 464 & 6166 & 1206756 \\
ring patterns $^{\mathrm{b}}$ & 24 & 32 & 144 & 208 \\
Riccati-type ring patterns $_{\text {distinct Riccati-type ring terms }}{ }^{\mathrm{c}}$ & 24 & 32 & 128 & 160 \\
\hline
\end{tabular}

a Germinal structure of the reference is assumed.

b Products of 2-body cumulants are omitted.

c Simplifications exploit integral, amplitude and cumulant symmetries and exchange of equivalent indices.

We presently do not aim at generating and implementing all terms at the MR CCD level. We rather wish to devise the simplest approximation to arrive at a MR rCCD theory paralleling the existing APSG based ERPA. First of all, terms at most linear in cumulants are kept only, since powers of cumulants do not appear in ERPA-APSG theory. Dropping terms including products of cumulants could be rationalized on the basis that their first power is already negligible. Unfortunately this argument is not always supported numerically.[40] This step is consequently better understood as a first approximation that may need rectification later.

As a second approximation, non-ring contraction patterns are neglected. Identification of ring patterns is performed based on the algebraic expression. In short, we consider a contraction pattern of ring type, if index pairs subject to the constraint introduced in Eq.(7) (e.g. $p<r$ ) can be introduced as hyper indices and the term can be recast as product of matrices, indexed by these hyper indices. There are some subtleties to this definition that are best conveyed by sketching the algorithm devised for the analysis. Let us consider a contraction pattern multiplied with the appropriate tensor elements (i.e. $f, v$ and $t$ ) according to Eq.(13). Summation is implied, indices not involved in summation are regarded outer. Among summation indices, constrained (originating from $T$ ) and unconstrained (originating from $H$ ) indices are distinguished. Prior to ring analysis, hyper indices (i.e. indices related by inequality) are established. We then go through the following steps, starting with the first contraction pattern

(1) reduce the number of summation indices by evaluating Kronecker-deltas implied by rank-1 contractions; favour constrained indices over unconstrained ones;

(2) if there are unconstrained summation indices still left, establish the tensor elements (i.e. $f, v$ or $\lambda$ ) they connect; tensor elements multiplied then summed for common, unconstrained indices are considered hyper tensors;

(3) initialize logical variable ring as 'true';

(4) take each hyper index formed of outer indices and determine whether the contributing orbital indices occur on the same tensor (i.e. $f, v, \lambda$ or $t$ ) or hyper tensor; if they occur on $t$, they even have to be arranged vertically; if any of this fails set ring 'false'

(5) if ring is 'true', goto 7

(6) if the expression is linear in $T$ and three of the outer indices occur on $t$ and one hyper index can be formed on $t$ vertically, the diagonal part of the hyper tensor is kept; otherwise the term is dropped; goto 9

(7) take each remaining hyper index and determine whether the contributing orbital 
indices occur on the same tensor (i.e. $f, v, \lambda$ or $t$ ) or hyper tensor; if they occur on $t$, they even have to be arranged vertically; if any of this fails set ring 'false';

(8) if ring is 'true', the term is kept, otherwise dropped;

(9) proceed to next contraction pattern and goto 1

When performed at the SR level, the above algorithm properly generates the ring CCD equations.

Let us consider some examples to illustrate the steps of ring filtering. Take e.g. the following two terms contributing to $\left\langle 0\left|\left\{E_{x y}^{u v}\right\}\left\{H_{1}\right\}\left\{T_{2}\right\}\right| 0\right\rangle, u<x, v<y$ :

$$
\begin{array}{rc}
-n_{u} n_{v} \bar{n}_{x} & \sum_{\substack{q r \\
q<x, u<r}} \alpha_{y q}^{v r} t_{r x}^{u q} \\
n_{u} n_{v} \bar{n}_{x} & \sum_{q<s}^{v s} \alpha_{y q}^{u q} t_{x s}^{q q}
\end{array}
$$

with the hyper tensor element

$$
\alpha_{y q}^{v r}=\sum_{t} f_{t}^{v} \Lambda_{y q}^{t r}
$$

The term in Eq.(14) is dropped at point 4, since outer indices $u$ and $x$, forming a hyper index, occur crosswise on amplitude $t$. The term of Eq.(15) is kept at point 4, since both index pairs $u x$ and $v y$ conform to the requirements. The expression of Eq.(15) is approved at point 7 also, where hyper index $q s$ is examined. It eventually contributes a term in the second row of the expression of $\boldsymbol{A}_{\mathrm{CC}}$, given in Appendix B.

An example for the diagonal term under point 6 , is given by the following expression, contributing to $\left\langle 0\left|\left\{E_{x y}^{u v}\right\}\left\{H_{2}\right\}\left\{T_{2}\right\}\right| 0\right\rangle, u<x, v<y$ :

$$
n_{u} n_{v} \bar{n}_{x} \bar{n}_{y} \sum_{\substack{r \\ u<r}} \beta_{x}^{r} t_{r y}^{u v}
$$

with the hyper tensor element

$$
\beta_{x}^{r}=\sum_{p q s} v_{x s}^{p q} \Lambda_{p q}^{s r}
$$

When making the ring approximation, $r \neq x$ terms of the sum in Eq.(16) are dropped. The $r=x$ term contributes a term in the last row of the expression of $\boldsymbol{A}_{\mathrm{CC}}$, given in Appendix B.

As a final example consider the following term contributing to $\left\langle 0\left|\left\{E_{x y}^{u v}\right\}\left\{H_{2}\right\}\left\{T_{2}^{2}\right\}\right| 0\right\rangle, u<x, v<y$ :

$$
-n_{u} \bar{n}_{x} \sum_{\substack{p<r \\ q<s \\ t<w}} n_{p} n_{q} \bar{n}_{s} \bar{n}_{w} v_{s w}^{p q} \Lambda_{y t}^{v r} t_{r s}^{p q} t_{w x}^{t u} .
$$

This is dropped at point 7 of the ring filtering as hyper index $p r$ is broken up, $p$ and $r$ occurring separately on tensors $v$ and $\Lambda$. The same hold for $t w$.

Eliminating non-ring terms reduces the number of contraction patterns considerably, as reflected in the third row of Table 1. One further trimming is still to be 
performed though. It turns out that not all terms admitted by the ring approximation conform with the algebraic Riccati equation derived from RPA equations. An example is provided by the following expression, again contributing to $\left\langle 0\left|\left\{E_{x y}^{u v}\right\}\left\{H_{2}\right\}\left\{T_{2}\right\}\right| 0\right\rangle$ :

$$
n_{v} \bar{n}_{y} \sum_{\substack{p<r \\ q<s}} \Lambda_{x p}^{u r} t_{r s}^{p q} n_{q} \bar{n}_{s} v_{q y}^{s v} .
$$

A characteristic feature of the term above is that tensor elements $\Lambda$ and $v$ are connected via $t$. When introducing hyper indices, the corresponding term involves two matrix factors multiplying $\boldsymbol{t}$, yielding an expression like $\boldsymbol{\Lambda} \boldsymbol{t} \boldsymbol{w}$ (with $w_{q s, v y}=n_{q} \bar{n}_{s} v_{q y}^{s v}$ ). This and similar terms are dropped presently as they can not be accommodated in the Riccati equation we wish to obtain. The remaining number of contraction patterns and the number of distinct terms upon performing simplifications are indicated in the last two rows of Table 1.

Adopting matrix notation, the final form of the Riccati-type ring amplitude equation is obtained as

$$
\boldsymbol{B}_{\mathrm{CC}}+2 \boldsymbol{A}_{\mathrm{CC}}^{T} \boldsymbol{t}+2 \boldsymbol{t} \boldsymbol{A}_{\mathrm{CC}}+4 \boldsymbol{t} \boldsymbol{M} \boldsymbol{B}_{\mathrm{CC}} \boldsymbol{M} \boldsymbol{t}=0
$$

with $t_{p r, q s}=t_{r s}^{p q}$ and subscript CC labeling matrix factors originating from the rCCD equations. Diagonal matrix $\boldsymbol{M}$ is built of occupation numbers as

$$
M_{p r, q s}=\delta_{p q} \delta_{r s} n_{p} \bar{n}_{r} .
$$

Elements of matrices $\boldsymbol{A}_{\mathrm{CC}}$ and $\boldsymbol{B}_{\mathrm{CC}}$ are given in Appendix B.

Amplitudes obtained from Eq.(17) are substituted in Eq.(11) to yield the MR rCCD energy correction, reading as

$$
\Delta E_{\mathrm{rCCD}}=\operatorname{Tr}\left(\boldsymbol{M} \boldsymbol{B}_{\mathrm{CC}} \boldsymbol{M} \boldsymbol{t}\right),
$$

neglecting terms including products of cumulants.

\section{Extended RPA based on APSG}

In the framework of equation of motion theory[24] an excitation operator of the companion RPA to the ring CCD of the previous Section is written as

$$
O_{\omega}^{\dagger}=\sum_{p<r}\left(X_{p r}^{\omega}\left\{E_{p}^{r}\right\}-Y_{p r}^{\omega}\left\{E_{r}^{p}\right\}\right)
$$

Regarding that

$$
\left\{E_{p}^{r}\right\}=E_{p}^{r}-\delta_{p r} n_{p}
$$

and that $p=r$ is excluded, the excitation operator of Eq.(20) agrees with that of extended RPA, c.f. Eq.(21) of Ref.[25]. Equations of ERPA determining excited state 
coefficients $X_{p r}^{\omega}, Y_{p r}^{\omega}$ and excitation energies $\omega$ are concisely written as

$$
\left(\begin{array}{rr}
A & B \\
-B & -A
\end{array}\right)\left(\begin{array}{c}
X \\
Y
\end{array}\right)=\left(\begin{array}{cc}
S & 0 \\
0 & S
\end{array}\right)\left(\begin{array}{c}
X \\
Y
\end{array}\right) \omega^{\mathrm{ERPA}}
$$

with

$$
\begin{aligned}
A_{q s, p r} & = & \left\langle 0\left|\left[E_{s}^{q},\left[H, E_{p}^{r}\right]\right]\right| 0\right\rangle / 2 \\
B_{q s, p r} & = & -\left\langle 0\left|\left[E_{s}^{q},\left[H, E_{r}^{p}\right]\right]\right| 0\right\rangle / 2 \\
S_{q s, p r} & = & \left\langle 0\left|\left[E_{s}^{q}, E_{p}^{r}\right]\right| 0\right\rangle / 2
\end{aligned}
$$

and $\omega$ being the column index in matrices $\boldsymbol{X}$ and $\boldsymbol{Y}$. Diagonal matrix $\boldsymbol{\omega}^{\mathrm{ERPA}}$ involves only the physically relevant, positive roots. (Factor $1 / 2$ in the above definition of matrices $\boldsymbol{A}, \boldsymbol{B}$ and $\boldsymbol{S}$ is introduced to agree with the expressions of Ref.[26]. The reversed sign of $\boldsymbol{B}$ originates in the reversed sign of $Y_{p r}^{\omega}$ in Eq.(20), compared with Ref.[25]. )

The overlap matrix on the rhs of Eq.(21) is diagonal, reading as

$$
S_{q s, p r}=\delta_{q p} \delta_{s r}\left(n_{p}-n_{r}\right)
$$

It is easy to see based on Eq.(2) that $\boldsymbol{S}$ can be decomposed as

$$
S=K L
$$

with $K_{q s, p r}=\delta_{q p} \delta_{s r}\left(c_{p}+c_{r}\right)$ and $L_{q s, p r}=\delta_{q p} \delta_{s r}\left(c_{p}-c_{r}\right)$. Supposing that $c_{p}+c_{r}$ and $c_{p}-c_{r}$ are nonzero for all $p, r$, Eq.(21) can be rewritten as

$$
\left(\begin{array}{rr}
\boldsymbol{A}^{-+} & \boldsymbol{B}^{--} \\
-\boldsymbol{B}^{++} & -\boldsymbol{A}^{+-}
\end{array}\right)\left(\begin{array}{c}
\boldsymbol{K} \boldsymbol{X} \\
\boldsymbol{L} \boldsymbol{Y}
\end{array}\right)=\left(\begin{array}{c}
\boldsymbol{K} \boldsymbol{X} \\
\boldsymbol{L} \boldsymbol{Y}
\end{array}\right) \omega^{\mathrm{ERPA}}
$$

with

$$
\begin{aligned}
& \boldsymbol{A}^{-+}=\boldsymbol{L}^{-1} \boldsymbol{A} \boldsymbol{K}^{-1} \\
& \boldsymbol{A}^{+-}=\boldsymbol{K}^{-1} \boldsymbol{A} \boldsymbol{K}^{-1} \\
& \boldsymbol{B}^{--}=\boldsymbol{L}^{-1} \boldsymbol{B} \boldsymbol{L}^{-1} \\
& \boldsymbol{B}^{++}=\boldsymbol{K}^{-1} \boldsymbol{B} \boldsymbol{K}^{-1}
\end{aligned}
$$

The Riccati-type equation associated with the ERPA equations can now be obtained by (i) multiplying the first equation in Eq. (22) by $(\boldsymbol{K} \boldsymbol{X})^{-1}$ from the right; (ii) multiplying the second equation in Eq.(22) by $(\boldsymbol{K} \boldsymbol{X})^{-1}$ from the right and left and subtracting the latter from the former. This yields

$$
\boldsymbol{B}^{++}+\boldsymbol{A}^{+-} \boldsymbol{C}_{R}+\boldsymbol{C}_{R} \boldsymbol{A}^{-+}+\boldsymbol{C}_{R} \boldsymbol{B}^{--} \boldsymbol{C}_{R}=0
$$

with

$$
\boldsymbol{C}_{R}=\boldsymbol{L} \boldsymbol{Y} \boldsymbol{X}^{-1} \boldsymbol{K}^{-1}
$$


subscript $R$ referring to the Riccati equation. When $\boldsymbol{K} \boldsymbol{X}$ is invertible, Eq.(23) is equivalent[41] with the ERPA equation of Eq.(21). Introduction of matrix factors $\boldsymbol{K}$ and $\boldsymbol{L}$ here is not completely coherent with Pernal[23], but it does not affect the essential equivalence with Eq.(21). Expression of matrix factors $\boldsymbol{A}^{+-}, \boldsymbol{A}^{-+}$and $\boldsymbol{B}^{++/--}$ is given in Appendix $\mathrm{C}$ to assist the comparison of the $\mathrm{rCCD}$ rooted Riccati equation Eq.(17) with Eq.(23), originating from ERPA. Note, that these equations are not exactly the same already at the SR level, there appears a factor of two between the solutions, c.f. Eq.(A10) of Appendix A. At the MR level the deviation is increased. While the SR limiting case of matrix factors in Eqs.(17) and (23) matches, the cumulant involving terms do not lend themselves to setting an explicit relation. An important difference concerns symmetry conservation of an initial guess during the iterative solution of Eqs.(17) and (23). Due to the appearance of $\boldsymbol{A}_{\mathrm{CC}}$ and its transpose in the linear terms and due to $\boldsymbol{B}_{\mathrm{CC}}$ being symmetric, symmetry of an initial $\boldsymbol{t}$ is not destroyed by the ring CCD equation. ${ }^{2}$ In the case of Eq.(23), symmetry of $\boldsymbol{C}_{R}$ is not ensured as $\boldsymbol{B}$ is not symmetric and $\boldsymbol{A}^{-+} \neq\left(\boldsymbol{A}^{+-}\right)^{T}$. A further difference between Eqs.(17) and (23) may be worth to note here. Matrices $\boldsymbol{K}$ or $\boldsymbol{L}$ becoming singular is a problem with Eq.(23), a caveat on $c_{p} \pm c_{r}$ getting close to zero has been in fact mentioned.[26] The Riccati equation in Eq.(17) could run into a similar problem if particle and hole occupation numbers appearing under the inverse in the expression of $\boldsymbol{A}_{\mathrm{CC}}$ and $\boldsymbol{B}_{\mathrm{CC}}$ became zero. This is however excluded by the restriction imposed on indices in Eq.(7) and Eq.(12).

It is possible to deduce an energy expression from the first equation of Eq.(22) by multiplying again from the right by $(\boldsymbol{K} \boldsymbol{X})^{-1}$ and performing a trace. This, so-called plasmon formula reads

$$
\Delta E_{\text {plasmon }}=\frac{1}{2} \operatorname{Tr}\left(\boldsymbol{\omega}^{\mathrm{ERPA}}-\boldsymbol{A}^{-+}\right)=\frac{1}{2} \operatorname{Tr}\left(\boldsymbol{B}^{--} \boldsymbol{C}_{R}\right) .
$$

This expression could be compared to the MR rCCD energy correction Eq.(19) were the relation between $\boldsymbol{C}_{R}$ and $\boldsymbol{t}$ known. At the SR level $\Delta E_{\text {plasmon }}$ agrees with $\Delta E_{\mathrm{rCCD}}$, c.f. Eq.(A19). This is the reason behind introducing the factor 1/2 in Eq.(25). To assist comparison between the SR and MR case, quantities introduced during the derivation and their relation are summarized in Tables 2 and 3and, respectively. At difference with the SR situation, there appears no obvious formal correspondence between the solutions of the Riccati equations Eq.(17)) and Eq.(23). For this reason a numerical comparison is called for contrasting the above $\Delta E_{\text {plasmon }}$ with $\Delta E_{\mathrm{rCCD}}$ at the $\mathrm{MR}$ level.

\section{Ground state consistent with ERPA}

Though Eq.(25) could be considered an ERPA energy correction formula, the analogous expression at the SR level (c.f Eqs.(A17) and (A18)) does not match the energy correction of the consistent ground state (c.f. Eq.(A16)) and the latter is preferred to the former in such a case.[42, 43] Motivated by this, we parametrize a ground state in

${ }^{2}$ This is at difference with the spin-orbital based formulation. [33] 
the ERPA framework as

$$
|\Phi\rangle=\left\{\exp \left(\frac{1}{2} \sum_{\substack{p<r \\ q<s}} \tau_{r s}^{p q}\left\{E_{p q}^{r s}\right\}\right)\right\}|0\rangle
$$

and seek an expression for amplitudes $\tau$ from the consistency condition

$$
O_{\omega}|\Phi\rangle=0
$$

Substituting $O_{\omega}$ from Eq.(20) we obtain

$$
\begin{aligned}
& \sum_{u<x}\left(X_{u x}^{\omega}\left\{E_{x}^{u}\right\}-Y_{u x}^{\omega}\left\{E_{u}^{x}\right\}\right)|0\rangle \\
+ & \frac{1}{2} \sum_{\substack{u<x \\
p<r, q<s}} \tau_{r s}^{p q}\left(X_{u x}^{\omega}\left\{E_{x}^{u}\right\}-Y_{u x}^{\omega}\left\{E_{u}^{x}\right\}\right)\left\{E_{p q}^{r s}\right\}|0\rangle=0 .
\end{aligned}
$$

Applying GWT, the second term on the lhs can be written as a sum of normal ordered products. Terms of the resulting expression can be identified as genuine 3-body, 2body etc. excitations out of APSG. In analogy with the SR treatment, at most single excitations are retained as a first approximation. A further simplification is introduced by neglecting terms involving cumulants of APSG. Among others, this latter step wipes all terms involving the unexcited APSG function, leading to

$$
\begin{aligned}
0= & \sum_{u<x}\left(X_{u x}^{\omega}\left\{E_{x}^{u}\right\}-Y_{u x}^{\omega}\left\{E_{u}^{x}\right\}\right)|0\rangle \\
+ & \frac{1}{2} \sum_{\substack{u<x \\
p<r, q<s}} \tau_{r s}^{p q} X_{u x}^{\omega} \\
& \left(2 \delta_{u p} \delta_{x r} n_{p} \bar{n}_{r}\left\{E_{q}^{s}\right\}+2 \delta_{u q} \delta_{x s} n_{q} \bar{n}_{s}\left\{E_{p}^{r}\right\}-\delta_{u p} \delta_{x s} n_{p} \bar{n}_{s}\left\{E_{q}^{r}\right\}-\delta_{u q} \delta_{x r} n_{q} \bar{n}_{r}\left\{E_{p}^{s}\right\}\right)|0\rangle \\
- & \frac{1}{2} \sum_{\substack{u<x \\
p<r, q<s}} \tau_{r s}^{p q} Y_{u x}^{\omega} \\
& \left(2 \delta_{u r} \delta_{x p} n_{p} \bar{n}_{r}\left\{E_{q}^{s}\right\}+2 \delta_{u s} \delta_{x q} n_{q} \bar{n}_{s}\left\{E_{p}^{r}\right\}-\delta_{u s} \delta_{x p} n_{p} \bar{n}_{s}\left\{E_{q}^{r}\right\}-\delta_{u r} \delta_{x q} n_{q} \bar{n}_{r}\left\{E_{p}^{s}\right\}\right)|0\rangle
\end{aligned}
$$

Index restrictions disclose the first two terms in the parenthesis multiplying $Y_{u x}^{\omega}$. Furthermore, we have to distinguish cases in the third and fourth term of the parenthesis multiplying $X_{u x}^{\omega}$, namely $q<r, q=r, r<q$ and $p<s, p=s, s<p$ respectively ${ }^{3}$.

${ }^{3}$ For simplicity, an ordering in decreasing order of $n_{p}$ is assumed in this section and $p<s$ is to be understood strictly, at difference with the more flexible requirement under Eq.(7). 
Upon simplifications, we are lead to

$$
\begin{aligned}
0 & =\sum_{\substack{p<s \\
q}} n_{p} \bar{n}_{s} \tau_{q s}^{p q} X_{p s}^{\omega}\left\{E_{q}^{q}\right\}|0\rangle \\
& +\sum_{p<r}\left(X_{p r}^{\omega}-\sum_{q<s} n_{q} \bar{n}_{s} \tau_{p s}^{q r} X_{q s}^{\omega}\right)\left\{E_{r}^{p}\right\}|0\rangle \\
& +\sum_{p<r}\left(-Y_{p r}^{\omega}+\sum_{q<s} n_{q} \bar{n}_{s}\left(2 \tau_{s r}^{q p}-\tau_{r s}^{q p}\right) X_{q s}^{\omega}+\sum_{q<s} n_{s} \bar{n}_{q} \tau_{r q}^{s p} Y_{q s}^{\omega}\right)\left\{E_{p}^{r}\right\}|0\rangle
\end{aligned}
$$

Note, that in the interest of transparency some index restrictions implied by amplitudes, are not indicated. These are $q<p, r<s$ in the second row above, and $p<q, s<r$ in the last term of the third row. One can proceed now by setting the terms of Eq.(26) zero individually. (This is consistent with requiring all projections with $\langle 0|\left\{E_{x}^{u}\right\}$ vanish, and neglecting the cumulant arising from e.g. $\left\langle 0\left|\left\{E_{x}^{u}\right\}\left\{E_{p}^{r}\right\}\right| 0\right\rangle$ here also.)

From the first row of Eq.(26) it then follows that spectator amplitudes are zero:

$$
\tau_{q s}^{p q}=0 \quad, \forall p<s, \forall q .
$$

From the second row, amplitudes with the following particular ordering of indices are zeroed

$$
\tau_{p s}^{q r}=0 \quad, \forall q<p<r<s .
$$

Using symmetry of the amplitude matrix $\boldsymbol{\tau}$, it follows from Eq.(27) that

$$
\tau_{r q}^{s p}=0 \quad, \forall p<q<s<r
$$

can be substituted in the third term of the third row of Eq.(26). A third consistency condition on the amplitudes is obtained consequently as

$$
\sum_{q<s} n_{q} \bar{n}_{s}\left(2 \tau_{s r}^{q p}-\tau_{r s}^{q p}\right) X_{q s}^{\omega}=Y_{p r}^{\omega}
$$

Introducing

$$
\left(C_{c}\right)_{r s}^{p q}=2 \tau_{r s}^{p q}-\tau_{s r}^{p q} \quad, \forall p, q<r, s
$$

matrix $\boldsymbol{C}_{c}$ stemming from the consistency condition can be written as

$$
\boldsymbol{C}_{c}=\boldsymbol{Y} \boldsymbol{X}^{-1} \boldsymbol{M}^{-1},
$$

admitting $p<r$ pairs for which $n_{p} \bar{n}_{r}$ is nonzero and assuming the existence of $\boldsymbol{X}^{-1}$. Note, that Eq.(29) determines only those elements of $\boldsymbol{C}_{c}$, where the indices conform with the restriction in Eq.(28). All other elements of $\boldsymbol{C}_{c}$ as well as the related amplitudes $\tau$ are zero.

It is interesting to observe, that the above, simplest condition for the consistent ground state at the MR level can not set all single excitations zero. The first term 
of the round bracket in the second row of Eq.(26) can not be eliminated within the approximations introduced. To get a refined picture, cumulants should be admitted in the consistency considerations.

For the time being we remain at the simplest level and observe the analogy of Eqs.(28) and (29) with their SR counterpart Eqs.(A15) and (Eq.(A8)). The expressions agree but for the diagonal matrix factor $\boldsymbol{M}^{-1}$ and the index restriction in Eq.(28) taking the place of occupied/virtual categorization. It is also interesting to notice, that $\boldsymbol{C}_{R}$ of Eq.(24), stemming fom the Riccati equation is not completely the same as $\boldsymbol{C}_{c}$ originating from the consistency condition. They differ not only by matrix factors built of occupation numbers but also in the number of zero entries. The latter follows from index restriction of Eq.(28) affecting $\boldsymbol{C}_{c}$ but not $\boldsymbol{C}_{R}$.

Deducing amplitudes $\tau$ from $\boldsymbol{C}_{c}$ as

$$
\tau_{r s}^{p q}=\frac{1}{3}\left(2\left(C_{c}\right)_{r s}^{p q}+\left(C_{c}\right)_{s r}^{p q}\right)
$$

an energy of the consistent ground state with ERPA can be obtained by substituting $\tau$ in the MR CCD energy formula of Eq.(19). Numerical comparison of this MR CCD energy expression with the plasmon formula of Eq.(25) would be desirable. For this end, implementation by computer based code generation is currently in progress in our laboratory.

\section{Conclusion}

The formulae worked out above set the previously designed ERPA into a CC context at the MR level. Rather loose approximations are introduced in the $\mathrm{CC}$ derivation to achieve close correspondence with SR expressions and allow for transparent comparison with ERPA. Neglect of cumulants altogether when obtaining a consistent ground state for ERPA appears to be the least justified approximation. Omission of powers of cumulants when deriving the ring equations is also to be tested numerically.

Explicit expressions are obtained at the MR level for the rCCD energy, the plasmon formula as well as the energy of the ground state consistent with ERPA. Tables 2 and 3 summarize in a concise manner the quantities introduced at the SR and MR level, respectively. Matching of the RPA and $\mathrm{rCCD}$ rooted Riccati equations allows to draw a formal relation between the energy expressions collected in Table 2 . At the MR level there appears no simple relation between matrix factors of the rCCD and ERPA rooted Riccati equations. For this reason a numerical comparison of the correlation energies will be necessary, with the ERPA-APSG energy formula[26] included as a fourth option.

The CC and ERPA formulae derived are general in the sense that explicit use of the reference being an APSG function was not made. It has to be kept in mind however, that omission of 3-body and higher cumulants (up to the number of electrons) follows from the geminal structure while it has to be regarded as an approximation for a non geminal reference. Taking APSG as reference is expected to have a beneficial effect on computational cost also, as all terms of the APSG cumulant is factorizable for Kronecker deltas, c.f. Eq.(3). The formal scaling of SR rCCD theory is therefore expected to persist, the MR extension affecting only the prefactor.

Let us comment finally on the superficial simplicity of the MR CC framework adopted here. As it was already alluded to, it has to be recognized that an enormous 
Table 2. Quantities introduced in a spin-free formulation of RPA and rCCD, based on the HF determinant. Matrix factors of the Riccati equation are given in Eqs.(A5) and (A6). See Eq.(A8) for the relation between $\boldsymbol{C}$ and $\boldsymbol{X}, \boldsymbol{Y}$, Eq.(A10) gives the relation between $\boldsymbol{C}$ and $\boldsymbol{t}$ and finally Eq.(A15) shows the relation between $\boldsymbol{C}$ and $\boldsymbol{\tau}$. For the relation of the energy expressions, see Eqs.(A17), (A18) and (A19). In particular $\Delta E_{\text {plasmon }}=\Delta E_{\mathrm{rCCD}}$.

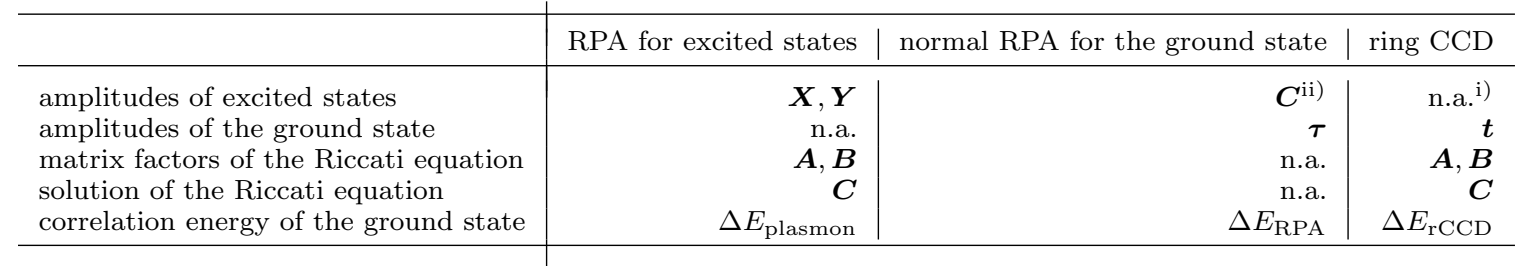

i) n.a.: not applicable

ii) A quantity derived from the amplitudes of excited states.

Table 3. Quantities introduced in a spin-free formulation of RPA and rCCD, based on a multideterminantal reference. Matrix factors of the Riccati equations are given Appendices B and C. See Eq.(24) for the relation between $\boldsymbol{C}_{R}$ and $\boldsymbol{X}, \boldsymbol{Y}$, Eq.(29) gives the relation between $\boldsymbol{C}_{c}$ and $\boldsymbol{X}, \boldsymbol{Y}$ and finally Eq.(28) shows the relation between $\boldsymbol{C}_{c}$ and $\boldsymbol{\tau}$.

\begin{tabular}{|c|c|c|c|}
\hline & RPA for excited states & normal RPA for the ground state & ring $\mathrm{CCD}$ \\
\hline amplitudes of the ground state & n.a. & $\tau$ & \\
\hline solution of the Riccati equation & $\boldsymbol{C}_{R}$ & n.a. & \\
\hline correlation energy of the ground state & $\Delta E_{\text {plasmon }}$ & $\Delta E_{\mathrm{RPA}}$ & $\Delta E_{\mathrm{rCCD}}$ \\
\hline
\end{tabular}

i) n.a.: not applicable

ii) A quantity derived from the amplitudes of excited states.

number of terms are generated by the GWT. In addition, there appears a redundancy among excited functions in the general case that has to be dealt with.[9] Redundancy is avoided here by resorting to double excitations in the cluster amplitudes. Extensivity is always an issue to consider, especially for a CC theory. While the expressions reported above appear connected at first glance, spin cumulants represent a non trivial problem $[44,45]$ and for this reason the analysis is put off to further study.

\section{Acknowledgement(s)}

Discussions with prof. P.R. Surján (Budapest) are gratefully acknowledged.

\section{Funding}

The work has been supported by the National Research, Development and Innovation Office (NKFIH), under grant number K115744. One of the authors (Á.Sz.) acknowledges support by the János Bolyai Research Scholarship of the Hungarian Academy of Sciences.

\section{References}

[1] K. Kowalski, P.D. Fan and P. Piecuch, Mol. Phys. 103, 2191-2213 (2005).

[2] I. Lindgren and D. Mukherjee, Physics Reports 151, 93 - 127 (1987).

[3] D. Mukherjee and S. Pal, Adv. Quantum Chem. 20, 291-373 (1989). 
[4] D. Sinha, S.K. Mukhopadhyay, R. Chaudhuri and D. Mukherjee, Chem. Phys. Letters 154, 544 (1989).

[5] R. Chaudhuri, D. Mukhopadhyay and D. Mukherjee, Chem. Phys. Letters 162, 393-398 (1989).

[6] B. Jeziorski and H.J. Monkhorst, Phys. Rev. A 24, 1668 (1981).

[7] U. Mahapatra, B. Datta and D. Mukherjee, J. Chem. Phys. 110, 6171-6188 (1999).

[8] U.S. Mahapatra, B. Datta, B. Bandyopadhyay and D. Mukherjee, in , Advances in Quantum Chemistry, Vol. 30, pp. 163 - 193.

[9] D. Sinha, R. Maitra and D. Mukherjee, Computational and Theoretical Chemistry 1003, $62-70$ (2013).

[10] D. Mukherjee, Chemical Physics Letters 274, 561 - 566 (1997).

[11] W. Kutzelnigg and D. Mukherjee, The Journal of Chemical Physics 107, 432-449 (1997).

[12] J. Č́žzek, J. Chem. Phys. 45, 4256 (1966).

[13] N. Ostlund and M. Karplus, Chemical Physics Letters 11, 450 - 453 (1971).

[14] A.E. Hansen and T.D. Bouman, Molecular Physics 37, 1713-1724 (1979).

[15] G.E. Scuseria, T.M. Henderson and D.C. Sorensen, The Journal of Chemical Physics 129, 231101 (2008).

[16] J. Oddershede, in Advances in Chemical Physics (John Wiley \& Sons, Inc., New York, 1987), Vol. 69, pp. 201-239.

[17] H. Eshuis, J.E. Bates and F. Furche, Theoretical Chemistry Accounts 131, 1084 (2012).

[18] G. Jansen, R.F. Liu and J.G. Ángyán, The Journal of Chemical Physics 133, $154106(2010)$.

[19] X. Ren, P. Rinke, C. Joas and M. Scheffler, Journal of Materials Science 47, 7447-7471 (2012).

[20] D. Peng, S.N. Steinmann, H. van Aggelen and W. Yang, The Journal of Chemical Physics 139, 104112 (2013).

[21] G.E. Scuseria, T.M. Henderson and I.W. Bulik, The Journal of Chemical Physics 139, 104113 (2013).

[22] W. Klopper, A.M. Teale, S. Coriani, T.B. Pedersen and T. Helgaker, Chemical Physics Letters 510, 147 - 153 (2011).

[23] K. Chatterjee and K. Pernal, The Journal of Chemical Physics 137, 204109 (2012).

[24] D. Rowe, Rev. Mod. Phys 40, 153 (1968).

[25] K. Pernal, Journal of Chemical Theory and Computation 10, 4332-4341 (2014).

[26] E. Pastorczak and K. Pernal, Phys. Chem. Chem. Phys. 17, 8622-8626 (2015).

[27] P.R. Surján, Topics in Current Chemistry 203, 63-88 (1999).

[28] P. Jeszenszki, P.R. Nagy, T. Zoboki, Á, Szabados and P.R. Surján, Int. J. Quantum Chem. 114, 1048 (2014).

[29] P.R. Surján, P. Jeszenszki and Á. Szabados, Molecular Physics 113, 2960-2963 (2015).

[30] W. Kutzelnigg, K. Shamasundar and D. Mukherjee, Molecular Physics 108, 433$451(2010)$.

[31] L. Kong, M. Nooijen and D. Mukherjee, The Journal of Chemical Physics 132, 234107 (2010).

[32] I. Lindgren, International Journal of Quantum Chemistry 14, 33-58 (1978).

[33] J. Toulouse, W. Zhu, A. Savin, G. Jansen and J.G. Ángyán, The Journal of 
Chemical Physics 135, 084119 (2011).

[34] L. Bytautas, T.M. Henderson, C.A. Jiménez-Hoyos, J.K. Ellis and G.E. Scuseria, The Journal of Chemical Physics 135, 044119 (2011).

[35] I. Shavitt and R.J. Bartlett, Many-Body Methods in Chemistry and Physics (Cambridge University Press, Cambridge, 2009).

[36] G. E. Scuseria and C. L. Janssen and H. F. Schaefer, J. Chem. Phys. 89, 7382 (1988).

[37] M. Hanauer and A. Köhn, Chemical Physics 401, 50 - 61 (2012), Recent advances in electron correlation methods and applications.

[38] R. Maitra, D. Sinha and D. Mukherjee, The Journal of Chemical Physics 137, 024105 (2012).

[39] L. Kong, K.R. Shamasundar, O. Demel and M. Nooijen, The Journal of Chemical Physics 130, 114101 (2009).

[40] J.M. Herbert, International Journal of Quantum Chemistry 107, 703-711 (2007).

[41] K. Zhou, J.C. Doyle and K. Glover, Robust and Optimal Control (Prentice-Hall, Inc., Upper Saddle River, NJ, USA, 1996).

[42] T.I. Shibuya and V. McKoy, Phys. Rev. A 2, 2208-2218 (1970).

[43] A. Szabo and N.S. Ostlund, The Journal of Chemical Physics 67, 4351-4360 (1977).

[44] R. Maitra, D. Sinha, S. Sen and D. Mukherjee, Theoretical Chemistry Accounts 133, 1522 (2014).

[45] A. Sen, S. Sen and D. Mukherjee, Journal of Chemical Theory and Computation 11, 4129-4145 (2015).

[46] F. Furche, The Journal of Chemical Physics 129, 114105 (2008).

[47] A.D. McLachlan and M.A. Ball, Rev. Mod. Phys. 36, 844-855 (1964).

\section{Appendix A. Relation between $E$-operator based RPA and rCCD}

In single reference theory, formulated with spin-orbitals, rCCD amplitude equations are equivalent to the RPA equations provided that $\boldsymbol{X}$ is invertible.[13, 15] The relation between $\mathrm{rCCD}$ and RPA amplitudes reads

$$
\boldsymbol{t}=\boldsymbol{Y} \boldsymbol{X}^{-1} .
$$

In addition, the rCCD wavefunction fulfills the killer condition of RPA up to 2-body excitations.[13] Accordingly, the $\mathrm{rCCD}$ wavefunction can be regarded as the ground state consistent with RPA excited states. The rCCD correlation energy is related to single excitation energies in the following simple manner $[13,15]$

$$
\Delta E=\frac{1}{4} \operatorname{Tr}\left(\boldsymbol{\omega}^{\mathrm{RPA}}-\boldsymbol{\omega}^{\mathrm{TDA}}\right),
$$

where $\boldsymbol{\omega}$ is the diagonal matrix of positive excitation energies, the superscript refers to the random phase and Tamm-Dancoff approximation (TDA) respectively and the Hartree-Fock reference is implied in the GNO. Relation Eq.(A1) can be exploited to set up an iterative cycle in RPA, redefining matrices $\boldsymbol{A}$ and $\boldsymbol{B}$ with the consistent ground state and extracting a new set of $\boldsymbol{X}$ and $\boldsymbol{Y}$. This procedure yields self-consistent RPA whereas we speak of normal RPA when $\boldsymbol{t}$ is extracted from $\boldsymbol{X}$ and $\boldsymbol{Y}$ but neither $\boldsymbol{A}, \boldsymbol{B}$ nor $\boldsymbol{X}, \boldsymbol{Y}$ are updated.[43] 
In this section we examine the relation between normal RPA and rCCD when both are formulated with the unitary generators of Eq.(6). We start from RPA and construct an excitation operator for solely singlet states

$$
O_{\omega}^{\dagger}=\sum_{i a}^{\prime}\left(X_{i a}^{\omega} E_{i}^{a}-Y_{i a}^{\omega} E_{a}^{i}\right)
$$

Indices $i, j, \ldots$ are assumed to be occupied and $a, b, \ldots$ are used for virtual orbitals in this section. For brevity primes on summation symbols indicate this restriction. Equations determining $\boldsymbol{X}$ and $\boldsymbol{Y} \operatorname{read}[42]$

$$
\left(\begin{array}{rr}
\boldsymbol{A} & \boldsymbol{B} \\
-\boldsymbol{B} & -\boldsymbol{A}
\end{array}\right)\left(\begin{array}{c}
\boldsymbol{X} \\
\boldsymbol{Y}
\end{array}\right)=\left(\begin{array}{c}
\boldsymbol{X} \\
\boldsymbol{Y}
\end{array}\right) \boldsymbol{\omega}^{\mathrm{RPA}}
$$

with

$$
\begin{aligned}
A_{j b, i a} & =\delta_{i j} \delta_{a b}\left(\varepsilon_{a}-\varepsilon_{i}\right)+\bar{v}_{j a}^{b i} \\
B_{j b, i a} & =\bar{v}_{j i}^{b a} .
\end{aligned}
$$

Provided that $\boldsymbol{X}^{-1}$ exists, the Riccati-equation associated with the eigenvalue problem Eq.(A4) reads

$$
B+A C+C A+C B C=0
$$

with

$$
C=\boldsymbol{Y} \boldsymbol{X}^{-1}
$$

Let us compare at this point Eq.(A7) to the Riccati-equation derived from the CCD equations formulated with unitary generators[36]. This involves making the ring approximation, leading to

$$
\boldsymbol{B}+2 \boldsymbol{A} \boldsymbol{t}+2 \boldsymbol{t} \boldsymbol{A}+4 \boldsymbol{t} \boldsymbol{B} \boldsymbol{t}=0 .
$$

The correspondence between rCCD equations Eq.(A9) and the RPA-associated Riccati-equation Eq.(A7) is close but not one to one, the solutions differ by a factor of 2

$$
C=2 t \text {. }
$$

Let us examine now the relation of $\boldsymbol{C}$ to the amplitude matrix $\boldsymbol{\tau}$ of the consistent ground state, c.f. normal RPA. For this end a parametrization of the double excitation 
operator is assumed $\mathrm{as}^{4}$

$$
T_{2}=\frac{1}{2} \sum_{i j a b}^{\prime} \tau_{i j}^{a b} E_{i j}^{a b}
$$

Let us suppose now, that

$$
|\Phi\rangle=e^{T_{2}}|\mathrm{HF}\rangle
$$

fulfills the killer condition

$$
O_{\omega}|\Phi\rangle=0
$$

Collecting terms including triple and higher excitations under $\mathcal{O}(3)$, Eq.(A13) leads to

$$
\sum_{i a}^{\prime}\left[Y_{i a}^{\omega *}-\sum_{j b}^{\prime} C_{a b}^{i j} X_{j b}^{\omega *}\right] E_{i}^{a}|\mathrm{HF}\rangle+\mathcal{O}(3)=0
$$

with

$$
C_{a b}^{i j}=2 \tau_{a b}^{i j}-\tau_{b a}^{i j}
$$

Neglecting $\mathcal{O}(3)$ terms, Eq.(A14) results Eq.(A8) for $\boldsymbol{C}$ accommodating $C_{a b}^{i j}$. Equations (A15) and (A8) represent the E-operator based counterpart of Eq.(A1). (Note, that amplitudes $\tau$ of the consistent ground state differ from rCCD amplitudes $t$. They can be related via Eqs.(A10) and (A15).) According to Eqs.(A15) and (A8), the Riccati-equation in Eq.(A7) is not written directly for the amplitudes of the consistent ground state. Having Eq.(A7) solved, amplitudes in Eq.(A12) can be inferred from Eq.(A15). (Amplitudes $\tau_{a b}^{i j}$ and $\tau_{b a}^{i j}$ are unrelated if $a \neq b$ and $i \neq j$ and the same holds for the elements of $\boldsymbol{C}$.)

Utilizing

$$
\tau_{a b}^{i j}=\frac{1}{3}\left(2 C_{a b}^{i j}+C_{b a}^{i j}\right)
$$

correlation energy of the consistent ground state (i.e. normal RPA) is expressed as

$$
\Delta E_{\mathrm{RPA}}=\operatorname{Tr}(\boldsymbol{B} \boldsymbol{\tau})=\operatorname{Tr}(\boldsymbol{v} \boldsymbol{)}
$$

with $v_{j b, i a}=v_{j i}^{b a}$.

We study finally the trace of excitation energy differences, the so-called plasmon

\footnotetext{
${ }^{4}$ The parametrization of Eq.(A11) is equivalent with the parametrization of Shibuya and McKoy[42] of the consistent ground state. Equations for $\tau$ however differ as they admit triplet excitations in $O_{\omega}^{\dagger}$ besides singlets and utilize the $S=0$ as well as the $S=1$ block of the RPA equations. Here we consider the $S=0$ block only.
} 
formula[46, 47] expressible from the first equation of Eq.(A4). This leads to

$$
\begin{aligned}
\Delta E_{\mathrm{plasmon}} & =\frac{1}{2} \operatorname{Tr}\left(\boldsymbol{\omega}^{\mathrm{RPA}}-\boldsymbol{\omega}^{\mathrm{TDA}}\right)=\frac{1}{2} \operatorname{Tr}(\boldsymbol{B C}) \\
& =\Delta E_{\mathrm{RPA}}-\frac{1}{2} \sum_{i j a b}^{\prime} v_{i j}^{b a} C_{a b}^{i j} \\
& =\Delta E_{\mathrm{RPA}}-\frac{1}{2} \sum_{i j a b}^{\prime} \bar{v}_{i j}^{b a} \tau_{a b}^{i j},
\end{aligned}
$$

a relation somewhat more complicated than Eq.(A2).

Approaching from the CC perspective, amplitudes $\boldsymbol{t}$ obtained from Eq.(A9) generate the rCCD correlation energy

$$
\Delta E_{\mathrm{rCCD}}=\operatorname{Tr}(\boldsymbol{B} \boldsymbol{t})=\frac{1}{2} \operatorname{Tr}\left(\boldsymbol{\omega}^{\mathrm{RPA}}-\boldsymbol{\omega}^{\mathrm{TDA}}\right),
$$

reminiscent of Eq.(A2). Correlation energies $\Delta E_{\mathrm{rCCD}}$ and $\Delta E_{\mathrm{RPA}}$ can be related based on Eqs.(A17), (A18) and (A19).

\section{Appendix B. Matrix factors of the MR rCCD Riccati equation, Eq.(17)}

$$
\begin{aligned}
\left(B_{\mathrm{CC}}\right)_{q s, p r} & =\bar{v}_{q p}^{s r} \\
& +\frac{1}{2}\left(\bar{n}_{s} \bar{n}_{r}\right)^{-1} \sum_{t u} v_{q p}^{t u} \Lambda_{t u}^{s r}+\frac{1}{2}\left(n_{q} n_{p}\right)^{-1} \sum_{t u} v_{t u}^{s r} \Lambda_{q p}^{t u} \\
& +\frac{1}{2}\left(n_{p} \bar{n}_{r}\right)^{-1} \sum_{t u} \bar{v}_{q t}^{s u} \Lambda_{u p}^{t r}+\frac{1}{2}\left(n_{q} \bar{n}_{s}\right)^{-1} \sum_{t u} \bar{v}_{p t}^{r u} \Lambda_{u q}^{t s} \\
& -\frac{1}{2}\left(n_{p} \bar{n}_{s}\right)^{-1} \sum_{t u}\left(v_{q t}^{r u} \Lambda_{u p}^{t s}+v_{q t}^{u r} \Lambda_{u p}^{s t}\right)-\frac{1}{2}\left(n_{q} \bar{n}_{r}\right)^{-1} \sum_{t u}\left(v_{p t}^{s u} \Lambda_{u q}^{t r}+v_{p t}^{u s} \Lambda_{u q}^{r t}\right) \\
\left(A_{\mathrm{CC}}\right)_{q s, p r} & =\delta_{q p} \delta_{s r}\left(\bar{n}_{r} f_{r}^{r}-n_{p} f_{p}^{p}\right)+n_{q} \bar{n}_{s} \bar{v}_{q r}^{s p}+\frac{1}{2} \delta_{q p} \delta_{s r} \sum_{t u} f_{t}^{u}\left(n_{p}^{-1} \Lambda_{u p}^{t p}-\bar{n}_{r}^{-1} \Lambda_{u r}^{t r}\right) \\
& +\frac{1}{2} \sum_{u}\left[n_{p}^{-1} f_{r}^{u} \Lambda_{u q}^{p s}-\bar{n}_{r}^{-1} f_{u}^{p} \Lambda_{r q}^{u s}+\left(n_{p} \bar{n}_{r}\right)^{-1}\left(\bar{n}_{s} f_{u}^{s} \Lambda_{r q}^{p u}-n_{q} f_{q}^{u} \Lambda_{r u}^{p s}\right)\right] \\
& +\frac{1}{2} \sum_{t u}\left[n_{q} \bar{n}_{r}^{-1}\left(v_{q u}^{p t} \Lambda_{t r}^{u s}+v_{q u}^{t p} \Lambda_{t r}^{s u}\right)+\bar{n}_{s} n_{p}^{-1}\left(v_{r u}^{s t} \Lambda_{t q}^{u p}+v_{r u}^{t s} \Lambda_{q t}^{u p}\right)\right. \\
& \left.-v_{q r}^{u t} \Lambda_{u t}^{s p} n_{q} n_{p}^{-1}-v_{u t}^{s p} \Lambda_{q r}^{u t} \bar{n}_{s} \bar{n}_{r}^{-1}+\bar{v}_{r u}^{p t} \Lambda_{t q}^{u s}+n_{q} \bar{n}_{s}\left(n_{p} \bar{n}_{r}\right)^{-1} \bar{v}_{q u}^{s t} \Lambda_{t r}^{u p}\right]- \\
& -\delta_{q p} \delta_{s r} \frac{1}{2} \sum_{t u v}\left[v_{r t}^{v u} \Lambda_{v u}^{r t}+v_{t v}^{r u} \Lambda_{r u}^{t v}+v_{t v}^{p u} \Lambda_{p u}^{t v}+v_{p t}^{u v} \Lambda_{u v}^{p t}\right]
\end{aligned}
$$


Appendix C. Matrix factors of the ERPA rooted Riccati equation,

Eq.(23)

$$
\begin{aligned}
A_{q s, p r}= & \delta_{q p} f_{s}^{r}\left(n_{p}-n_{s}\right)+\delta_{s r} f_{p}^{q}\left(n_{r}-n_{q}\right)+\bar{v}_{s p}^{q r}\left(n_{s} n_{r}+n_{q} n_{p}-n_{q} n_{r}-n_{s} n_{p}\right) \\
+ & \frac{1}{4} \sum_{u x}\left(2 v_{s x}^{r u} \Lambda_{p u}^{q x}+2 v_{u p}^{x q} \Lambda_{s x}^{r u}+2 v_{s x}^{u r} \Lambda_{p u}^{x q}+2 v_{u p}^{q x} \Lambda_{s x}^{u r}\right. \\
& \left.-v_{p s}^{x u} \Lambda_{x u}^{r q}-v_{x u}^{q r} \Lambda_{s p}^{x u}-v_{p s}^{u x} \Lambda_{x u}^{q r}-v_{x u}^{r q} \Lambda_{s p}^{u x}\right) \\
- & \frac{1}{4} \sum_{u v x}\left[\delta_{q p}\left(v_{u v}^{r x} \Lambda_{s x}^{u v}+v_{u v}^{x r} \Lambda_{s x}^{v u}\right)+\delta_{s r}\left(v_{x p}^{u v} \Lambda_{u v}^{x q}+v_{x p}^{v u} \Lambda_{u v}^{q x}\right)\right] \\
\left(A^{-+}\right)_{q s, p r}= & \left(c_{q}-c_{s}\right)^{-1} A_{q s, p r}\left(c_{p}+c_{r}\right)^{-1} \\
\left(A^{+-}\right)_{q s, p r}= & \left(c_{q}+c_{s}\right)^{-1} A_{q s, p r}\left(c_{p}-c_{r}\right)^{-1} \\
B_{q s, p r}=-A_{q s, r p} & \left(c_{q} \pm c_{s}\right)^{-1} B_{q s, p r}\left(c_{p} \pm c_{r}\right)^{-1} \\
& \left(B^{++/--}\right)_{q s, p r}=(
\end{aligned}
$$

